\title{
PROGRAMA REDE MÃe PARANAENSE: ANÁlISE DA ATENÇÃO PRÉ-NATAL EM UMA REGIONAL DE SAÚDE
}

\author{
Maria Aparecida Baggio ${ }^{1}$, Fernanda de Castro Pereira ${ }^{2}$, Ana Tereza Bittencourt Guimarães ${ }^{3}$, Sebastião \\ Caldeira $^{4}$, Claudia Silveira Viera ${ }^{1}$
}

RESUMO: Estudo quantitativo, descritivo, retrospectivo, que objetivou analisar a atenção pré-natal em uma Regional de Saúde do Estado do Paraná, entre 2012 e 2013. Os dados foram coletados de relatórios mensais e anuais, com instrumento contendo indicadores de avaliação conforme matrizes do Programa Rede Mãe Paranaense. De acordo com o preconizado pelo Programa, os municípios da Regional de Saúde estudada obtiveram resultados insatisfatórios no que diz respeito ao início do pré-natal no primeiro trimestre e realização de testes rápidos de HIV e sífilis, visto que em sua totalidade foram inferiores a 70\%. Quanto aos registros e informações referentes à realização do pré-natal, identificou-se ausência de informações sobre a quantidade de gestantes imunizadas e que realizaram consultas odontológicas, e possível atraso de registro nos sistemas de informação. Considerando-se a implantação do Programa Rede Mãe Paranaense em 2012, sugere-se a realização de outros estudos para avaliar as ações de saúde no pré-natal e seus registros.

DESCRITORES: Cuidado pré-natal; Mortalidade materna; Atenção primária à saúde; Programas de saúde; Equipes de saúde.

\section{REDE MÃE PARANAENSE PROGRAM: ANALYSIS OF PRENATAL CARE IN A REGIONAL HEALTH DISTRICT}

ABSTRACT: Quantitative, descriptive and retrospective study to analyze prenatal care in a Regional Health District in the State of Paraná, Brazil, between 2012 and 2013. The data were collected from monthly and annual reports, using a tool with assessment indicators according to the matrices of the Rede Mãe Paranaense (Network of Mothers in the State of Paraná) Program. According to the program recommendations, the cities in the Regional Health District studied obtained unsatisfactory results concerning the start of prenatal care in the first term and the accomplishment of rapid HIV and syphilis tests, in view of total rates inferior to $70 \%$. Concerning the records and information on prenatal care practice, absence of information was found on the number of pregnant women who were immunized and went through dentral appointments, as well as a possible delay to register the data in the information systems. Concerning the implementation of the Rede Mãe Paranaense Program in 2012, other studies are suggested to assess the prenatal health care actions and their records.

DESCRIPTORS: Prenatal care; Maternal mortality; Primary health care; Health teams.

\section{PROGRAMA REDE MÃE PARANAENSE: ANÁlISIS DE LA ATENCIÓN PRENATAL EN UNA REGIONAL DE SALUD}

RESUMEN: Estudio cuantitativo, descriptivo, retrospectivo con objeto de analizar la atención prenatal en una Regional de Salud del Estado de Paraná entre 2012 y 2013. Los datos fueron recolectados de relatos mensuales y anuales, mediante instrumento con indicadores de evaluación según matrices del Programa Rede Mãe Paranaense (Red de Madres del Estado de Paraná). Según la recomendación del Programa, los municipios de la Regional de Salud estudiada alcanzaron resultados insatisfactorios respecto al inicio del prenatal en el primer trimestre y aplicación de pruebas rápidas de VIH y sífilis, ya que en su totalidad fueron inferiores al $70 \%$. Respecto a los registros e informaciones referentes a la práctica del prenatal, fue identificada ausencia de informaciones sobre la cuantidad de gestantes inmunizadas y que hicieron consultas odontológicas, y posible retraso de registro en los sistemas de información. Ante la implantación del Programa Rede Mãe Paranaense en 2012, se sugiere el desarrollo de otros estudios para evaluar las acciones de salude en el prenatal y sus registros.

DESCRIPTORES: Cuidado prenatal; Mortalidad materna; Atención primaria de salud; Programas de salud; Equipos de salud.

${ }^{1}$ Enfermeira. Doutora em Enfermagem. Docente da Universidade Estadual do Oeste do Paraná. Cascavel, PR, Brasil.

${ }^{2}$ Discente de Enfermagem. Universidade Estadual do Oeste do Paraná. Cascavel, PR, Brasil.

${ }^{3}$ Bióloga. Doutora em Ecologia e Recursos Naturais. Docente da Universidade Estadual do Oeste do Paraná. Cascavel, PR, Brasil.

${ }^{4}$ Enfermeiro. Doutor em Enfermagem. Docente da Universidade Estadual do Oeste do Paraná. Cascavel, PR, Brasil.

Autor Correspondente:

Fernanda de Castro Pereira

Universidade Estadual do Oeste do Paraná

R. Edson Beller de Oliveira, 829 - 85819776 - Cascavel, PR, Brasil

E-mail: fernanda.unioeste@gmail.com
Recebido: $19 / 02 / 2016$

Finalizado: 23/09/2016 


\section{INTRODUÇÃO}

A Razão de Mortalidade Materna (RMM) reflete a qualidade de atenção à saúde da mulher. Taxas elevadas representam insatisfatória atenção no planejamento familiar, pré-natal, parto e puerpério. No Brasil, em 1990 a RMM era de 140 óbitos/100 mil Nascidos Vivos (NV). Em 2011 essa razão declinou para 64 óbitos/100 mil NV, tendo redução de 55\% na taxa de mortalidade materna ${ }^{(1)}$. No Paraná, em 1990 a RMM era de 90,5/100 mil NV declinando em 2000 para 66,4/100 mil NV. No entanto, no período de 2001 a 2010 houve estagnação na redução desses índices, em 2001 a RMM era de 65,3/100 mil NV e em 2010 de 65,1/100 mil NV ${ }^{(2)}$. Esses índices estão aquém do preconizado pela Organização Pan-Americana da Saúde/Organização Mundial da Saúde (OPAS/OMS) como baixa RMM, que se refere a menos que 20 mortes/100 mil NV ${ }^{(2)}$. De acordo com o quinto Objetivo de Desenvolvimento do Milênio (ODM), o Brasil deveria apresentar RMM igual ou inferior a 35 óbitos/100 mil NV até 2015. Ao final de 2015 nota-se que o Brasil apresentou redução da mortalidade ${ }^{(1)}$, contudo este índice continua superior aos recomendados.

As causas de morte materna diretas resultam de complicações na gravidez, parto e puerpério, decorrentes de omissões, tratamento incorreto ou uma cadeia de eventos associados. As indiretas decorrem de doenças preexistentes ou desenvolvidas na gestação(1). No Paraná, as causas diretas mais frequentes são a Doença Hipertensiva Específica da Gestação (DHEG) e as hemorragias ${ }^{(2)}$.

A Atenção Primária à Saúde (APS) atua na prevenção da mortalidade materna por meio da captação precoce da gestante e ordenamento do cuidado gravídico-puerperal nos demais níveis de atenção ${ }^{(3)}$. No Paraná, a atenção pré-natal é conduzida pelas diretrizes do Programa Rede Mãe Paranaense (PRMP), fundamentado na Rede Cegonha, com objetivo de assegurar à mulher o direito ao planejamento reprodutivo e atenção humanizada à gravidez, ao parto e ao puerpério e à criança o direito ao nascimento seguro e ao crescimento e desenvolvimento saudáveis ${ }^{(2,4)}$.

O PRMP visa à implantação de ações e atenção materno-infantil, prevendo a captação precoce da gestante, o seu acompanhamento no pré-natal, com recomendação de no mínimo sete consultas, a realização de exames, a estratificação de risco das gestantes, com direcionamento a ambulatório especializado para as de risco e a garantia de parto conforme risco gestacional, com gestante vinculada ao hospital de acordo com a classificação de risco gestacional. Esse programa teve sua implantação no ano de 2012, abrangendo 339 municípios do Estado do Paraná, pertencentes a 22 Regionais de Saúde (RS), e prevê que a consulta e a solicitação de exames podem ser realizados por Médicos e Enfermeiros ${ }^{(2)}$.

Nesse contexto, questiona-se: como se encontra a atenção pré-natal na $10^{\mathrm{a}}$ Regional de Saúde (RS) do Paraná, após a implantação do PRMP? O estudo objetivou analisar a atenção pré-natal de gestantes pertencentes à $10^{a}$ RS do Paraná, no período de 2012 a 2013, a fim de apontar sugestões relevantes para aperfeiçoamento das ações do PRMP na atenção pré-natal.

\section{MÉTODO}

Estudo quantitativo, descritivo e retrospectivo ${ }^{(5)}$, desenvolvido na $10^{\mathrm{a}} \mathrm{RS}$, situada no município de Cascavel, Paraná. Os dados referem-se aos anos de 2012 e 2013, e a coleta foi realizada de agosto a dezembro de 2014, a partir de relatórios mensais e anuais de cada município da $10^{\mathrm{a}} \mathrm{RS}$. Foi elaborado instrumento de coleta, embasado nas matrizes do PRMP, com os indicadores de avaliação: Quantidade de gestantes cadastradas no Sistema de Informação em Saúde Pré-natal (SIS-Pré-natal) no município; de gestantes que iniciaram o pré-natal no primeiro trimestre; de gestantes com 7 ou mais consultas obstétricas; quantidade total de consultas obstétricas por gestante; de gestantes que realizaram consultas odontológicas; de gestantes imunizadas; de gestantes que realizaram teste rápido de gravidez, de sífilis, de HIV, de exames realizados por trimestre gestacional; e estratificação de risco das gestantes com referência para atendimento.

A partir dos dados, as variáveis quantitativas (avaliadas por municípios), foram descritas quanto a suas estatísticas descritivas (média, mínimo, máximo, desvio padrão). 
As variáveis que apresentaram registro no ano de 2013 (Quantidade de gestantes/1000 hab, Quantidade de gestantes com sete ou mais consultas, Quantidade total de consultas, Quantidade de gestantes que iniciaram o pré-natal no primeiro trimestre, Quantidade de gestantes que realizaram os exames do $1^{\circ}$ trimestre, do $2^{\circ}$ trimestre, do $3^{\circ}$ trimestre, de teste sífilis, de teste gravidez, de teste HIV, \% Partos normais, \% Cesárea, Índice de mortalidade) foram analisadas por meio da Análise de Agrupamento Hierárquica Aglomerativa (AHA), utilizando o método de aglomeração de Ward. A medida de distância utilizada para formação dos agrupamentos foi a de Bray-Curtis, uma estatística utilizada para classificar diferentes localidades, baseada nas variações das variáveis analisadas. Na AHA são formados grupos com base no padrão de variação de cada.

Após a definição dos agrupamentos, as variáveis foram comparadas entre os grupos, sendo previamente avaliadas quanto ao padrão de distribuição dos dados por meio do teste de normalidade de Shapiro-Wilk e a homogeneidade das variâncias, por meio do Teste de Levene. Os pressupostos de normalidade e homogeneidade das variâncias são exigidos para que sejam realizadas análises paramétricas. Como tais pressupostos não foram satisfeitos, as variáveis foram comparadas por meio do Teste não Paramétrico de Kruskal-Wallis, seguidos do teste de acompanhamento de Dunn, uma vez que fora realizada a comparação entre 3 agrupamentos. Em todas as análises estatísticas foi estabelecido um nível de significância de 0,05.

Este estudo contempla parte do projeto intitulado Análise do Processo de Implantação e Desenvolvimento do PRMP, aprovado em Edital Chamada Universal, do CNPq, número 474768/20139. Obteve-se parecer favorável número 544.107 em 27/03/2014, pelo Comitê de Ética em Pesquisa, da Universidade Estadual do Oeste do Paraná.

\section{RESUltADOS}

Sobre as variáveis Quantidade de gestantes cadastradas no SIS-Pré-natal, Quantidade de gestantes que iniciaram o pré-natal no $1^{\circ}$ trimestre e Quantidade de consultas obstétricas, o registro dessas informações iniciou apenas em 2013, não havendo informações referentes ao período anterior (2012). Sobre a variável Quantidade de consultas odontológicas, não há evidência de registro.

Quanto à variável quantidade de gestantes cadastradas no SIS-Pré-natal, no ano de 2013, identificouse que 58\% (n=14) dos municípios apresentaram 7 a 14 gestantes/1000 hab.; três (13\%) municípios apresentaram 14,1 a 17,5 gestantes/1000 hab.; sete (29\%) dos municípios apresentaram 0 a 7 gestantes/1000 hab cadastradas no SIS-Pré-natal. Um dos municípios da $10^{a}$ RS não possui registro da quantidade de gestantes (Figura 1).

Quanto ao início do pré-natal, evidenciou-se que em média $66 \%$ das gestantes o iniciaram ainda no primeiro trimestre. Em 13 municípios (52\%), mais de 70\% das mulheres iniciaram o pré-natal no primeiro trimestre. Porém, em 12 (48\%) municípios, menos de $70 \%$ das gestantes iniciaram o pré-natal no primeiro trimestre (Figura 2).

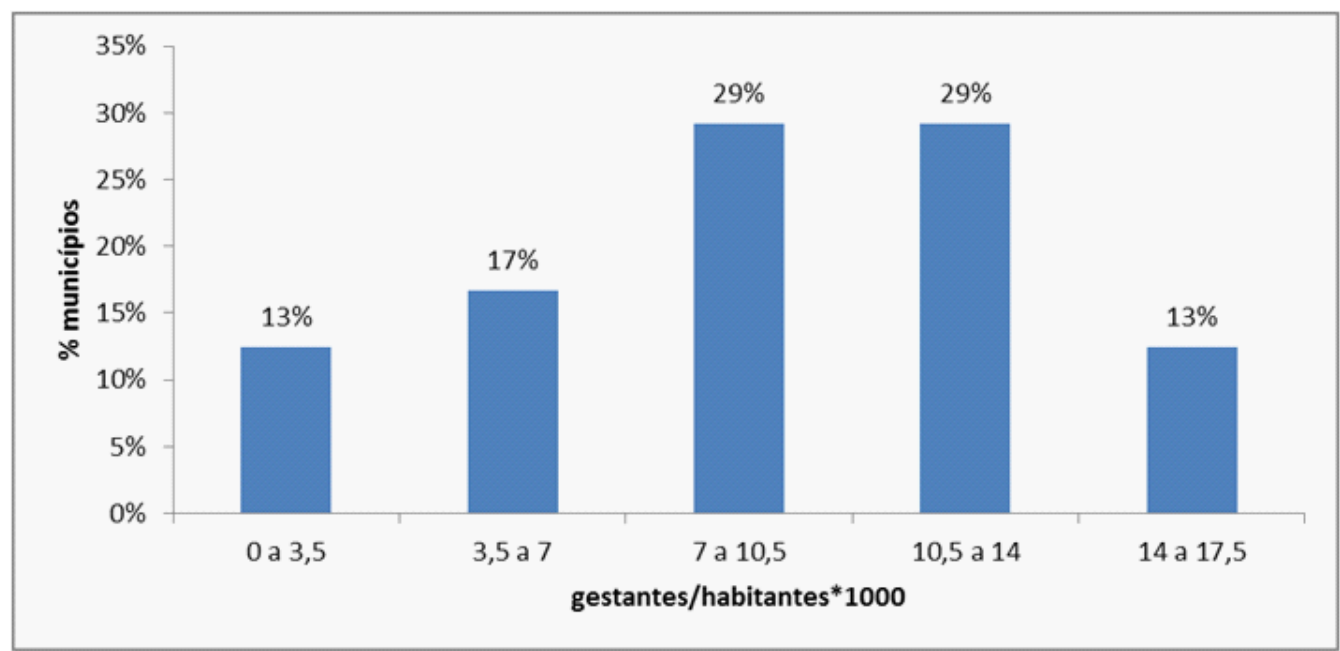

Figura 1 - Percentual de municípios em relação ao coeficiente de gestantes por 1000 habitantes. Cascavel, Paraná, Brasil, 2014 


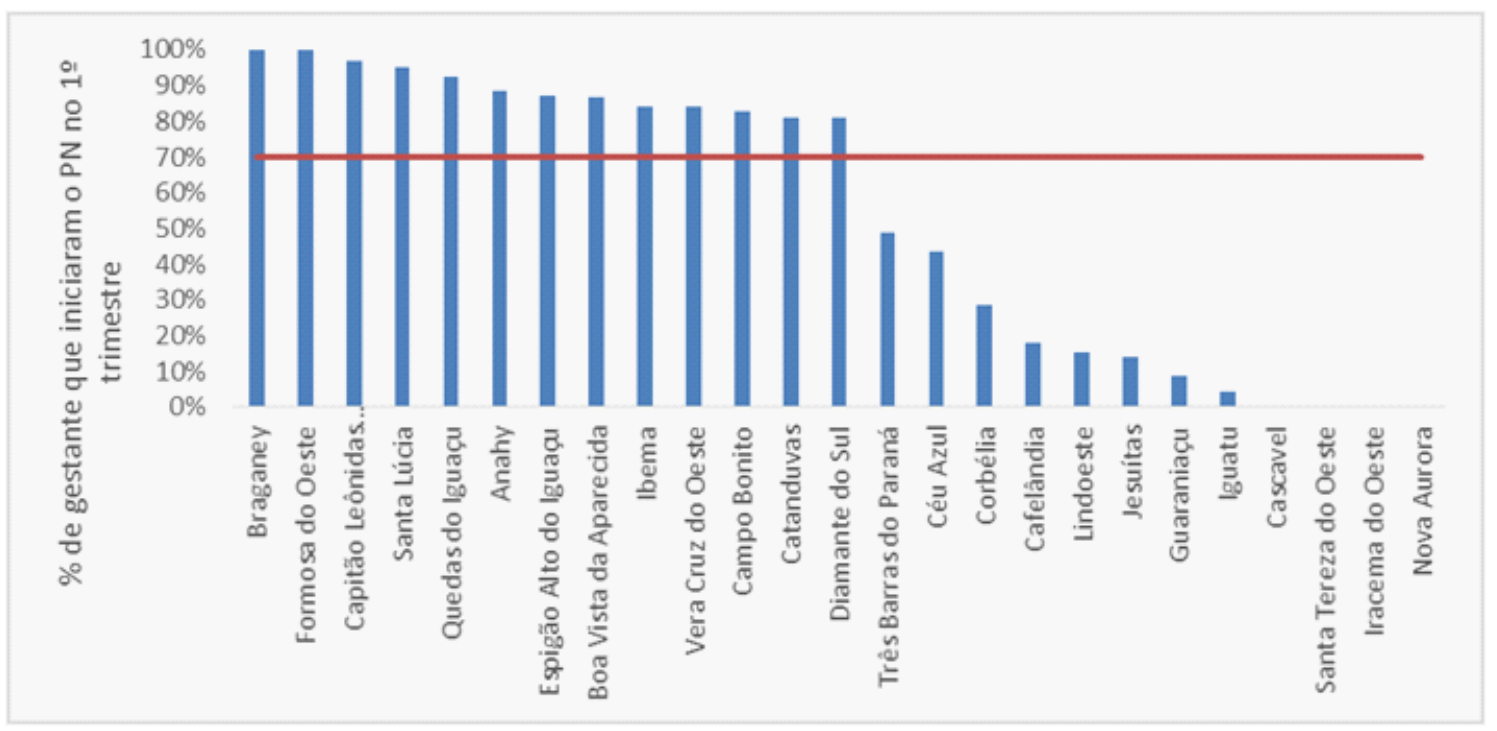

Figura 2 - Percentual de gestantes por município que iniciaram o pré-natal no primeiro trimestre. Cascavel, Paraná, Brasil, 2014

Quanto à cobertura pré-natal, em 2013, 18 (72\%) municípios apresentaram menos de 70\% das gestantes com sete consultas obstétricas ou mais. O município de Nova Aurora não apresenta registros sobre o número de consultas obstétricas. Os dados do município de Cascavel referem-se a apenas cinco gestantes, não havendo registro das demais (Figura 3). Identificou-se, em média, 3,6 consultas obstétricas por gestante. Salienta-se que o preconizado pelo PRMP é de seis consultas durante o período gestacional e uma no puerpério, totalizando minimamente sete consultas.

Referente à quantidade de gestantes imunizadas, não se evidenciou registro após a implantação do PRMP.

Após a apresentação do cenário geral relativo às gestantes, foi realizada análise de agrupamento objetivando demonstrar os municípios que apresentam semelhanças quanto às variáveis analisadas em 2013. Foi possível verificar o agrupamento de municípios em três classes: Grupo 1 - Anahy, Cafelândia, Campo Bonito, Céu Azul, Diamante do Sul, Espigão Alto do Iguaçu, Iguatu, Iracema do Oeste, Jesuítas, Lindoeste, Nova Aurora, Santa Lúcia, Santa Tereza do Oeste; Grupo 2 - Boa Vista da Aparecida, Capitão Leônidas Marques, Catanduvas, Corbélia, Guaraniaçu, Ibema, Quedas do Iguaçu, Três Barras do Paraná, Vera Cruz do Oeste; Grupo 3 - Braganey, Cascavel, Formosa do Oeste (Figura 4).

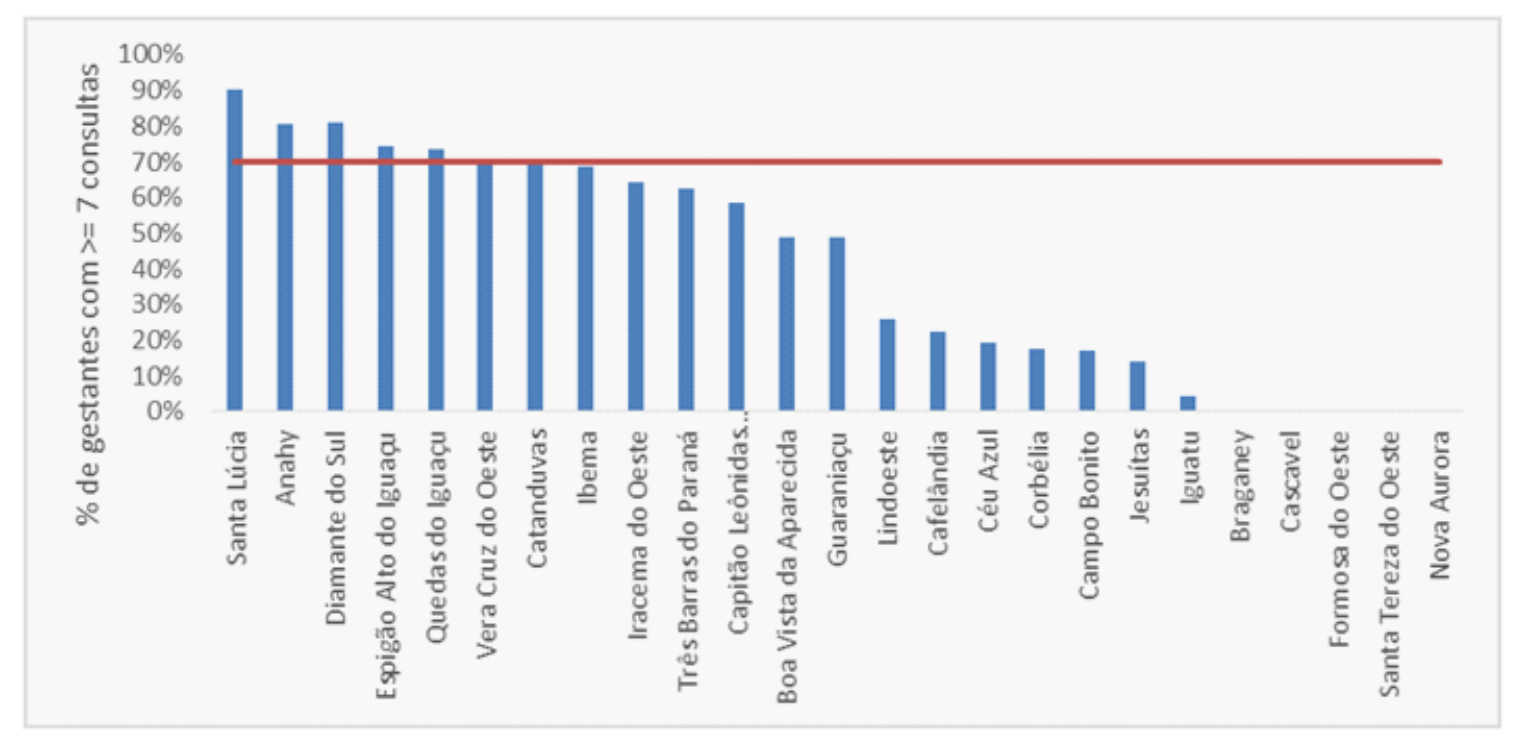

Figura 3 - Percentual de gestantes por município que realizaram 7 ou mais consultas durante o pré-natal. Cascavel, Paraná, Brasil, 2014 


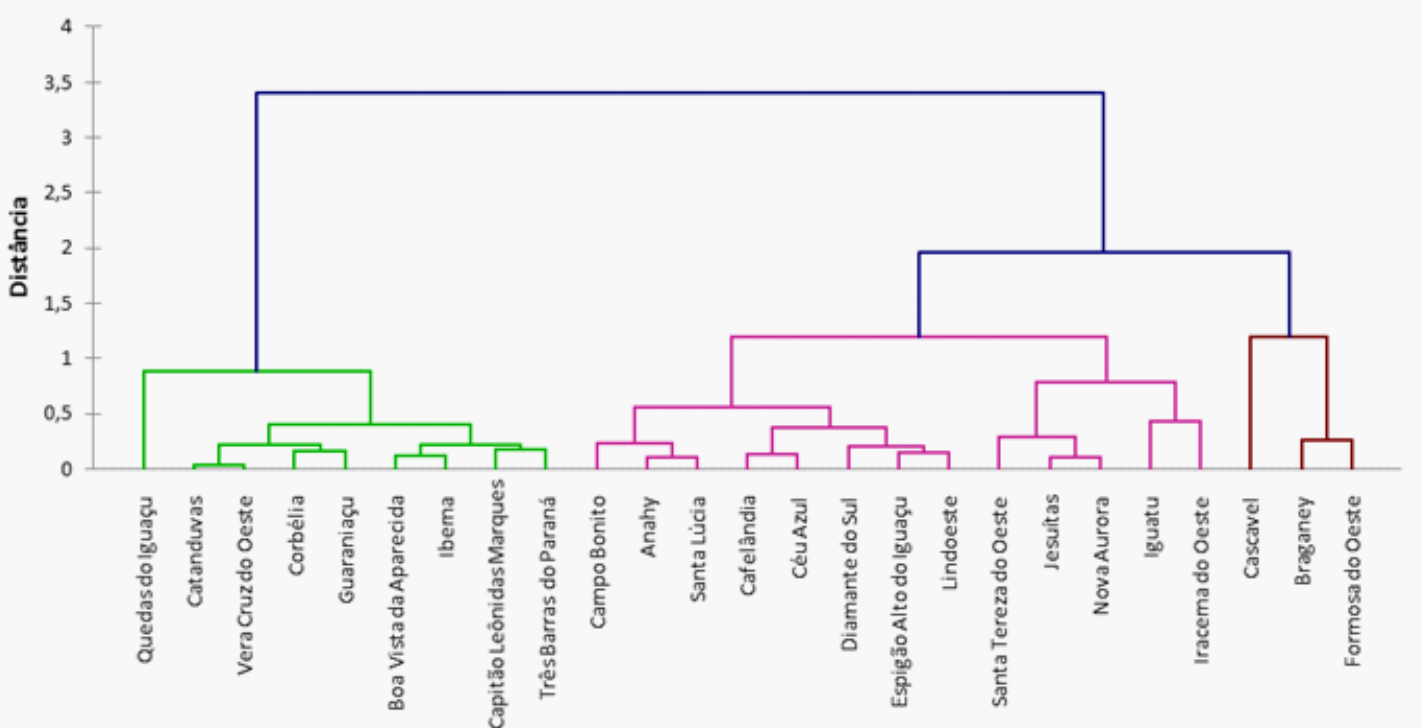

Figura 4 - Dendrograma de agrupamento dos municípios por meio do método de aglomeração de Ward e distância de Bray-Curtis. Cascavel, Paraná, Brasil, 2014

Estes grupos foram comparados entre si, para avaliar as suas particularidades, as quais estão demonstradas na Tabela 1.

Observou-se que o Grupo 2 apresentou os maiores valores relativos às variáveis estudadas, enquanto o Grupo 1 apresentou significativamente os menores valores $(p<0,05)$. O Grupo 3 apresentou uma discrepância na junção dos municípios no agrupamento. Nesse grupo, está o município de Cascavel, o qual apresentou registro de cinco gestantes em 2013. Nesse grupo encontram-se ainda os municípios de Braganey e Formosa do Oeste, que também apresentaram um baixo número de gestantes registradas. Contudo, assim como Cascavel, esses municípios apresentam um elevado número de partos. Tudo leva a crer que nestes municípios há falha nos registros nos sistemas de informação. Assim, a análise relatada será principalmente para os Grupos 1 e 2.

Tabela 1 - Estatísticas descritivas (Média + Desvio Padrão - DP) das variáveis analisadas nos três grupos de municípios da $10^{a}$ Regional de Saúde do Paraná, obtidos por meio da análise de agrupamento. Cascavel, Paraná, Brasil, 2014

\begin{tabular}{|c|c|c|c|}
\hline Variáveis (unidade) & Grupo 1 & Grupo 2 & Grupo 3 \\
\hline Habitantes (n) & $6815 \pm 4483^{b}$ & $13954 \pm 7878^{\mathrm{a}}$ & $107470 \pm 174756^{\mathrm{ab}}$ \\
\hline Gestantes (n) & $56+35^{a}$ & $170 a+88^{a}$ & $2+2^{b}$ \\
\hline Gestantes/1000 hab (n) & $9+4^{\mathrm{ab}}$ & $13+3^{a}$ & $0,1+0,1^{\mathrm{b}}$ \\
\hline Gestantes com $>7$ consultas $(n)$ & $17+12^{b}$ & $97+70^{a}$ & $0+0^{\mathrm{b}}$ \\
\hline Total de consultas de PN entre as Gestantes (n) & $157 \pm 68^{b}$ & $746 \pm 497^{a}$ & $3^{+} 4^{\mathrm{b}}$ \\
\hline Gestantes que iniciaram o $\mathrm{PN}$ no $1^{\circ}$ trimestre $(\mathrm{n})$ & $31+14^{\mathrm{b}}$ & $114+45^{\mathrm{a}}$ & $1+0^{\mathrm{b}}$ \\
\hline Gestantes imunizadas no $1^{\circ}$ trimestre $(\mathrm{n})$ & $20+15^{b}$ & $114+94^{a}$ & $1+1^{b}$ \\
\hline Gestantes imunizadas no $2^{\circ}$ trimestre $(n)$ & $5 \pm 6^{a}$ & $27 \pm 51^{\mathrm{a}}$ & $0,3 \pm 0,6^{a}$ \\
\hline Gestantes imunizadas no $3^{\circ}$ trimestre $(\mathrm{n})$ & $7+8^{b}$ & $55+75^{a}$ & $0,7+1,2^{b}$ \\
\hline Gestantes que realizaram Teste sífilis (n) & $7+10^{\mathrm{ab}}$ & $83+96^{a}$ & $0,3+0,6^{b}$ \\
\hline Gestantes que realizaram Teste gravidez $(\mathrm{n})$ & $0,2+0,6^{\mathrm{a}}$ & $2,1+5,6^{a}$ & $0,0+0,0^{\mathrm{a}}$ \\
\hline Gestantes que realizaram Teste HIV (n) & $7 \pm 10^{\mathrm{ab}}$ & $84 \pm 96^{a}$ & $0,3 \pm 0,6^{b}$ \\
\hline Partos normais (n) & $28+21^{\mathrm{b}}$ & $70+27^{a}$ & $602+1008^{\mathrm{ab}}$ \\
\hline Partos cesáreos (n) & $54+49^{a}$ & $104+76^{a}$ & $987+1614^{a}$ \\
\hline Índice mort. materna (n/100.000 nascidos vivos) & $0,0+0,0^{\mathrm{a}}$ & $0,4+0,7^{\mathrm{a}}$ & $0,3+0,6^{a}$ \\
\hline Municípios (n) & 13 & 9 & 3 \\
\hline
\end{tabular}


O Grupo 1 é composto por 13 municípios de pequeno a médio porte, apresentando menos de 20.000 habitantes (Anahy: 2.922 habitantes; Cafelândia: 16.321habitantes; Campo Bonito: 4.309 habitantes; Céu Azul: 11.589 habitantes; Diamante do Sul: 3.575 habitantes; Espigão Alto do Iguaçu: 4.591 habitantes; Iguatu: 2.300 habitantes; Iracema do Oeste: 2.537 habitantes; Jesuítas: 9.017 habitantes; Lindoeste: 5.247 habitantes; Nova Aurora: 11.659 habitantes; Santa Lúcia: 3.986 habitantes; Santa Tereza do Oeste: 10.528 habitantes). Em média, estes municípios tiveram 56 gestantes no ano de 2013, cujo coeficiente médio foi de 9,2 gestantes por 1000 habitantes. Sobre o tipo de parto, foram registrados, em média, $27,5+20,5$ partos normais e 53,5+48,5 cesáreas. Esses dados sugerem que gestantes de outros municípios pertencentes à RS estudada e ou de outras RS migraram para os referidos municípios.

Nesses locais, aproximadamente $55 \%(n=367)$ das gestantes iniciaram o pré-natal no primeiro trimestre, contudo, apenas $30 \%(n=198)$ das gestantes realizam sete ou mais consultas obstétricas durante a gestação $(16,5 \pm 11,7$ gestantes). Quanto à realização de exames, $36 \%(n=239)$ das gestantes fizeram os exames solicitados no $1^{\circ}$ trimestre $(19,9 \pm 15,0), 10 \%(n=64)$ realizaram os exames no $2^{\circ}$ trimestre $(5,3+6,3)$ e $13 \%(n=85)$ realizaram os exames no $3^{\circ}$ trimestre $(7,1 \pm 7,6)$. Sobre os testes rápidos, $12 \%(n=79)$ das gestantes fizeram o teste de sífilis, $(0,36 \%)$ duas fizeram teste de gravidez e $13 \%(n=85)$ teste de HIV. O índice de mortalidade materna foi zero.

O Grupo 2 é composto por nove municípios de médio porte, apresentando entre 6.000 a 33.000 habitantes (Boa Vista da Aparecida: 7.968 habitantes; Capitão Leônidas Marques: 15.724 habitantes; Catanduvas: 10.459 habitantes; Corbélia: 17.076 habitantes; Guaraniaçu: 13.998 habitantes; Ibema: 6.352 habitantes; Quedas do Iguaçu: 32.982 habitantes; Três Barras do Paraná: 12.227 habitantes; Vera Cruz do Oeste: 8.998 habitantes). Em média, estes municípios tiveram 170 gestantes no ano de 2013, cujo coeficiente médio foi de 12,7 gestantes por 1000 habitantes.

Sobre o tipo de parto, foram registrados $69,7 \pm 26,5$ partos normais e $104,4 \pm 75,9$ cesáreas, sugerindo que não houve migração das gestantes da $10^{\mathrm{a}} \mathrm{RS}$ para outros municípios desta ou de outra RS do Estado do Paraná. Nos municípios do Grupo 2, aproximadamente $67 \%(n=874)$ das gestantes iniciaram o prénatal no primeiro trimestre, sendo que $57 \%(n=1029)$ das gestantes realizam sete ou mais consultas obstétricas $(96,9 \pm 69,8$ gestantes). Quanto à realização de exames, $67 \%(n=1027)$ fizeram os exames solicitados no $1^{\circ}$ trimestre $(114,19 \pm 94,3), 16 \%(n=245)$ realizaram os exames no $2^{\circ}$ trimestre $(27,2 \pm 51,1)$ e $32 \%(n=494)$ realizaram os exames no $3^{\circ}$ trimestre $(54,9 \pm 74,9)$. Sobre os testes rápidos, $49 \%(n=747)$ das gestantes fizeram o teste de sífilis, 1,23\% $(n=19)$ fizeram teste de gravidez e $50 \%(n=758)$ teste de HIV. O índice de mortalidade materna no período estudado (2012-2013) foi 0,4 40,7 .

\section{DISCUSSÃO}

O estudo realizou breve avaliação da atenção pré-natal na $10^{\mathrm{a}}$ RS do Paraná, após a implantação do PRMP. Referente ao período de início do pré-natal, identificou-se média inferior a $70 \%$ de gestantes que o iniciaram no primeiro trimestre, cujo índice assemelha-se a outras regiões brasileiras ${ }^{(6-7)}$, como no município de Porto Alegre, RS, em que $52 \%$ das mulheres iniciaram o pré-natal no primeiro trimestre ${ }^{(7)}$.

Contraditoriamente a esses dados, estudos revelaram índices favoráveis quanto ao início do prénatal, os quais identificaram percentuais superiores a $70 \%$ de gestantes que o iniciaram no primeiro trimestre $^{(8-12)}$. Destes, estudo destaca que mais de $75 \%$ das gestantes iniciaram o pré-natal no primeiro trimestre, sendo que $18,2 \%$ das mulheres o iniciaram no primeiro ou segundo mês gestacional ${ }^{(13)}$.

A assistência pré-natal, desde o primeiro trimestre, é condição necessária para a promoção da saúde materna e infantil. Nesse sentido, o monitoramento dos fatores de risco, responsáveis por agravos à saúde, contribui favoravelmente quando detectados e tratados precocemente ${ }^{(10)}$.

O início do acompanhamento pré-natal está relacionado à qualidade da organização dos serviços de APS, acesso e vínculo da comunidade à unidade de saúde. Quando iniciado tardiamente, sinaliza fragilidade desta atenção. Assim, de acordo com o PRMP a equipe, e particularmente os Agentes Comunitários de Saúde, devem identificar precocemente as gestantes, priorizando aquelas de alto risco $^{(2)}$.

A deficiência na sensibilização das gestantes, a falta de informação, a dificuldade de realizar busca 
ativa e captar as gestantes precocemente ${ }^{(13)}$ podem justificar o início tardio do pré-natal identificado neste estudo.

Referente à cobertura do pré-natal, 18 dos 25 municípios apresentaram menos de $70 \%$ das gestantes com sete consultas obstétricas ou mais, sendo 3,6 consultas por gestante a média. Esses dados não correspondem à recomendação da Organização Mundial da Saúde $(\mathrm{OMS})^{(14)}$, do Programa de Humanização do Pré-natal e Nascimento (PHPN) do $\mathrm{MS}^{(15)}$, e preconizados pelo PRMP, sendo inferiores se comparados aos de outras realidades brasileiras ${ }^{(6-8)}$.

A OMS recomenda no mínimo, quatro consultas no período gestacional ${ }^{(14)}$. No Paraná, o PRMP segue as recomendações do MS, com uma consulta no primeiro trimestre gestacional, duas no segundo, três no terceiro trimestre e uma no puerpério(2). Indo ao encontro do que também é preconizado em países como os Estados Unidos, em que as consultas no pré-natal devem ocorrer mensalmente desde o início da gravidez até $28^{a}$ semana, quinzenalmente entre a $28^{a}$ e $36^{a}$ e semanalmente da $36^{a}$ semana até o parto ${ }^{(16)}$.

O percentual de mulheres que realizou quatro consultas de pré-natal, em países em desenvolvimento, apresentou aumento de 37\% em 1990 para cerca de 52\% em 2012. Em países de baixa renda, entre 2006 a 2013, apenas 38\% das gestantes realizaram quatro consultas de pré-natal. Mesmo que a OMS recomende a realização de no mínimo quatro consultas no pré-natal, entre 2006 e 2013 somente 56\% das gestantes ao redor do mundo atenderam ao recomendado ${ }^{(14)}$, demonstrando pouca melhora nesse indicador.

O número de consultas parece controverso ao esperado de um pré-natal de qualidade. Mesmo com número reduzido de consultas, o pré-natal de mulheres de baixo risco pode ser adequado se houver avaliação e maior atenção àquelas que apresentem maior fator de risco gestacional.

O número inadequado de consultas de pré-natal está relacionado a mães com baixa escolaridade e ao nascimento de bebês com baixo peso ${ }^{(17)}$. Isso vai ao encontro de outras experiências, as quais relacionam a mortalidade materna e infantil à escolaridade da mãe, às condições de vida e ao acesso aos serviços de saúde em tempo oportuno ${ }^{(2)}$.

A consulta odontológica está contemplada como parte do pré-natal no Brasil e também no Estado do Paraná. No entanto, neste estudo não foi evidenciado registro de consultas odontológicas por gestantes no período estudado. Estudo brasileiro demonstra que apenas $17 \%$ das gestantes receberam algum tipo de assistência odontológica ${ }^{(18)}$, evidenciando que esta não é uma realidade apenas local.

Durante a gestação, ocorre maior vascularização da cavidade oral devido à elevação nos níveis de progesterona e estrogênio, ocasionando o aumento da permeabilidade vascular e alterações na produção de colágeno, tornando a gengiva mais suscetível a infecções e ao biofilme bacteriano ${ }^{(19)}$. Essa condição também torna a gestante vulnerável a outros acometimentos, como diabetes mellitus gestacional $^{(20)}$. Ainda, a doença periodontal tem sido relacionada como fator de risco para o parto prematuro e baixo peso ao nascer ${ }^{(21)}$. Emerge então, a necessidade de promover a saúde bucal materna para se reduzir os riscos à gestante e seu filho no pré-natal.

Outro aspecto importante refere-se ao monitoramento da gestante e da criança. O SIS-Pré-natal (Sistema de Monitoramento e Avaliação do Pré-Natal, Parto, Puerpério e Criança) permite a realização de cadastro de gestantes e recém-nascidos, desde o diagnóstico de gravidez até o nascimento da criança. Assim, o sistema subsidia Municípios, Estados e o MS com informações que permitam aos gestores planejar, acompanhar e avaliar as ações de promoção, prevenção e assistência, a fim de reduzir taxas de morbimortalidade materna, perinatal e neonatal ${ }^{(22)}$.

A consistência dos achados deste estudo apresentou fragilidade, visto que a coleta dos dados, a partir do SIS-Pré-natal, denota incongruência entre número de habitantes e número de gestantes cadastradas no sistema. Exemplo disso é o município de Cascavel, PR, que, segundo dados do Instituto Brasileiro de Geografia e Estatística (IBGE) ${ }^{(23)}$, possui população superior a 300 mil habitantes, e, no entanto, apresentou cinco gestantes cadastradas em 2013. Logo, pode-se questionar se os profissionais de saúde estão cadastrando e registrando adequadamente as gestantes atendidas e alimentando o SIS-Pré-natal para acompanhamento do pré-natal e puerpério, ou se houve atraso no registro destas informações. É atribuição do profissional de saúde da atenção básica o cadastramento da gestante 
no início do pré-natal, bem como a realização da estratificação de risco e a vinculação da gestante ao serviço para o parto, conforme risco gestacional ${ }^{(2)}$.

Em revisão integrativa, apontou-se fragilidade na forma de organização e prática do Sistema de Informação em Saúde (SIS), comprometendo a fidedignidade dos dados produzidos, pois não representem a realidade da situação de saúde da população brasileira ${ }^{(24)}$.

Assim, existe a necessidade de conhecer o nível de confiabilidade dos SIS e a origem dos dados, visto que esses são imprescindíveis no que diz respeito à necessidade de mudança e adoção de novas estratégias. Faz-se necessário que a qualidade da informação seja objeto de estudos, sinalizando fragilidades, para que os reparos necessários sejam feitos, resultando em dados fidedignos ${ }^{(25)}$.

A realização de exames e testes rápidos no período gestacional para detecção de sífilis e HIV é de fundamental importância para garantir intervenção e tratamento adequados. Considera-se o tratamento da sífilis com especial importância, visto que a prevalência desta doença é indicador da qualidade da assistência, pois seu diagnóstico e tratamento são ofertados pela rede básica de saúde $^{(9)}$. Os tratamentos, sendo realizados de forma precoce e correta, podem reduzir as chances de transmissão vertical para apenas $8,3 \%{ }^{(26)}$. Para uma assistência pré-natal efetiva, o Programa Nacional de Imunizações (PNI), do MS, no período do estudo, recomendava imunização da gestante contra o tétano, influenza, hepatite $\mathrm{B}^{(27)}$. No entanto, não se identificou registro de gestantes imunizadas no pré-natal.

Quanto à estratificação de risco, salienta-se que o PRMP teve início em 2011, tendo com um dos objetivos a realização da estratificação de risco habitual, intermediário e alto risco das gestantes. A Rede Cegonha, segundo a Portaria $n^{0} 1.459$ de 24 de junho de 2011, do $M S^{(5)}$, desde 2011 prevê a realização do pré-natal, com solicitação de exames e vinculação da gestante ao serviço de saúde conforme o risco. No presente estudo, todos os municípios aderiram à estratificação de risco, o que mostra um dos aspectos positivos da implantação do PRMP.

Referente ao número de cesáreas, em detrimento do parto normal no Brasil, importa salientar que as práticas de parto e nascimento desde o século passado sofreram transformações decorrentes da mercantilização, medicalização e hospitalização do parto, indicando urgência no resgate natural de parir. A cesárea representou no ano de 2012 no Brasil, 52\% dos nascimentos, sendo 86\% no setor privado e $43 \%$ no público ${ }^{(28)}$, valores muito superiores aos $10 \%$ a $15 \%$ preconizados pela $\mathrm{OMS}^{(14)}$.

Além disso, o parto normal, em relação à cesárea, possui vantagens como menor custo ao sistema de saúde e propicia rápida recuperação da mulher ${ }^{(1)}$. Estudo revelou que os altos índices de cesáreas registrados atualmente relacionam-se com a influência cultural e familiar, o medo da dor, bem como o desejo da mulher em realizar a laqueadura tubária e, principalmente, a escassez de informações sobre benefícios e desvantagens das vias de parto ${ }^{(29)}$.

\section{CONSIDERAÇÕES FINAIS}

De acordo com o que preconiza o PRMP, os municípios da $10^{\mathrm{a}} \mathrm{RS}$ do Estado do Paraná obtiveram resultados insatisfatórios no que diz respeito ao início do pré-natal no primeiro trimestre de gestação e realização de testes rápidos de HIV e sífilis, visto que em sua totalidade foram inferiores a $70 \%$. Quanto aos registros e informações referentes à realização do pré-natal, identificou-se ausência de informações sobre a quantidade de gestantes imunizadas e que realizaram consultas odontológicas, e possível atraso de registro nos sistemas de informação.

As ações e estratégias em saúde de atenção pré-natal propostas pelo PRMP, no Estado do Paraná, a priori, na $10^{\mathrm{a}}$ RS estudada, necessitam de ampliação a fim de alcançar índices de adesão às estratégias próximas aos $100 \%$, garantindo melhor qualidade na assistência materna e infantil.

Considerando os dados obtidos e a implantação do PRMP em 2012, sugere-se a realização de outros estudos semelhantes a este para avaliar as ações de saúde voltadas à atenção pré-natal e seus registros no Estado do Paraná. 
1. Programa das Nações Unidas para o Desenvolvimento (PNUD). Objetivos de desenvolvimento para o milênio. Melhorar a saúde materna. [Internet] 2016 [acesso em 28 mar 2016]. Disponível:

http://www.pnud.org.br/ODM5.aspx.

2. Paraná. Secretaria de Estado da Saúde do Paraná (SESA). Programa Rede Mãe Paranaense: Linha guia. [Internet] Paraná: SESA; 2012 [acesso em 12 jan 2015]. Disponível:

http://www.saude.pr.gov.br/arquivos/File/MaeParanaense_2014_LinhaGuia_Ed03_148x210mm__1.pdf.

3. Duarte SJH, de Andrade SMO. Assistência pré-natal no Programa Saúde da Família. Esc. Anna Nery. [Internet] 2006; 10(1) [acesso 11 nov 2015]. Disponível: http://dx.doi.org/10.1590/S1414-81452006000100016.

4. Brasil. Portaria n. 1.459, de 24 de junho de 2011. Institui, no âmbito do Sistema Único de Saúde - SUS - a Rede Cegonha. Brasília: Ministério da Saúde; 2011.

5. Cervo AL, Bervian PA, da Silva R. Metodologia Científica. 6a ed. São Paulo: Pearson Prentice Hall; 2007.

6. Polgliane RBS, Leal MC, Amorim MHC, Zandonade E, dos Santos Neto ET. Adequação do processo de assistência pré-natal segundo critérios do Programa de Humanização do Pré-natal e Nascimento e da Organização Mundial de Saúde. Ciênc. saúde coletiva. [Internet] 2014; 19(7) [acesso em 07 nov 2015]. Disponível:

http://dx.doi.org/10.1590/1413-81232014197.08622013.

7. Hass CN, Teixeira LB, Beghetto MG. Adequabilidade da assistência pré-natal em uma estratégia de saúde da família de Porto Alegre - RS. Rev. Gaúcha Enferm. [Internet] 2013; 34(3) [acesso em 12 dez 2015]. Disponível: http://dx.doi.org/10.1590/S1983-14472013000300003.

8. Viellas EF, Domingues RMSM, Dias MAB, da Gama SGN, Theme Filha MM, da Costa JV, et al. Assistência prénatal no Brasil. Cad. Saúde Pública. [Internet] 2014; 30(Supl.1) [acesso em 12 dez 2015]. Disponível:

http://dx.doi.org/10.1590/0102-311X00126013.

9. de Oliveira RLA, da Fonseca CRB, Cavalhaes MABL, Parada CMGL. Evaluation of pre-natal care from the perspective of different models in primary care. Rev. Latino-Am. Enfermagem. [Internet] 2013; 21(2) [acesso em 08 dez 2015]. Disponível: http://dx.doi.org/10.1590/S0104-11692013000200011.

10. Domingues RMSM, Hartz ZMA, Dias MAB, Leal MC. Avaliação da adequação da assistência pré-natal na rede SUS do Município do Rio de Janeiro, Brasil. Cad. Saúde Pública. [Internet] 2012; 28(3) [acesso em 01 jan 2016]. Disponível: http://dx.doi.org/10.1590/S0102-311X2012000300003.

11. Corrêa MD, Tsunechiro MA, Lima MOP, Bonadio IC. Avaliação da assistência pré-natal em unidade com estratégia saúde da família. Rev. esc. enferm. USP. [Internet] 2014; 48(n.esp) [acesso em 01 jan 2016]. Disponível: http://dx.doi.org/10.1590/S0080-623420140000600004.

12. da Silva EP, de Lima RT, Ferreira NLS, Costa MJC. Pré-natal na atenção primária do município de João PessoaPB: caracterização de serviços e usuárias. Rev. Bras. Saúde Mater. Infant. [Internet] 2013; 13(1) [acesso em 20 nov 2015]. Disponível: http://dx.doi.org/10.1590/S1519-38292013000100004.

13. Vilarinho LM, Nogueira LT, Nagahama EEl. Avaliação da qualidade da atenção à saúde de adolescentes no pré-natal e puerpério. Esc. Anna Nery. [Internet] 2012; 16(2) [acesso em 09 dez 2015]. Disponível: http://dx.doi. org/10.1590/S1414-81452012000200015.

14. World Health Organization (WHO). Global Health Observatory. [Internet] Antenatal care [acesso em 22 jan 2015]. Disponível: http://www.who.int/gho/maternal_health/reproductive_health/antenatal_care_text/en/.

15. Ministério da Saúde (BR). Programa de Humanização do Pré-natal e Nascimento. Humanização no parto. [Internet] Brasília: Ministério da Saúde; 2002 [acesso em 12 fev 2015]. Disponível:

http://bvsms.saude.gov.br/bvs/publicacoes/parto.pdf.

16. Office on Woman's Health U.S. (US). Department of Health and Human Services, Office on Women's Health. Prenatal care fact sheet. [Internet] 2012 [acesso em 17 jan 2015]. Disponível:

http://www.womenshealth.gov/publications/our-publications/fact-sheet/prenatal-care.html. 
17. Gonçalves AC, Costa MCN, Braga JU. Análise da distribuição espacial da mortalidade neonatal e de fatores associados, em Salvador, Bahia, Brasil, no período 2000-2006. Cad. Saúde Pública. [Internet] 2011; 27(8) [acesso em 18 nov 2015]. Disponível: http://dx.doi.org/10.1590/S0102-311X2011000800013.

18. Santos Neto ET, Oliveira AE, Zandonade E, Leal MC. Acesso à assistência odontológica no acompanhamento pré-natal. Ciênc. saúde coletiva. [Internet] 2012;17(11) [acesso em 22 nov 2015]. Disponível:

http://dx.doi.org/10.1590/S1413-81232012001100022.

19. Zanata RL, Fernandes KBP, Navarro PSL. Prenatal dental care: evaluation of professional knowledge of obstetricians and dentists in the cities of Londrina/PR and Bauru/SP, Brazil, 2004. J. Appl. Oral Sci. [Internet] 2008; 16(3) [acesso em 22 nov 2015]. Disponível: http://dx.doi.org/10.1590/S1678-77572008000300006.

20. Chokwiriyachit A, Dasanayake AP, Suwannarong W, Hormdee D, Sumanonta G, Prasertchareonsuk W, et al. Periodontitis and gestational diabetes mellitus in non-smoking females. J Periodontol. [Internet] 2013; 84(7) [acesso em 22 nov 2015].

21. Rodrigues AS, Lima DBGO, Ganhito JA, Romito GA, Lotufo RFM, Micheli G, et al. Parto prematuro e baixo peso ao nascer associados à doença periodontal: aspectos clínicos, microbiológicos e imunológicos. Rev. odontol. Univ. Cid. Sao Paulo. 2004; 16(1): 55-61.

22. Ministério da Saúde (BR). Datasus. SIS Pré Natal. [Internet] Brasília: Ministério da Saúde; 2015 [acesso em 18 fev 2015]. Disponível: http://datasus.saude.gov.br/sistemas-e-aplicativos/epidemiologicos/sisprenatal.

23. Instituto Brasileiro de Geografia e Estatística (IBGE). Cidades. Cascavel. [Internet] 2015 [acesso em 18 fev 2015]. Disponível: http://cidades.ibge.gov.br/xtras/perfil.php?lang=\&codmun=410480.

24. dos Santos SR, Ferreira JA, Cruz EMMS, Leite EMAM, Pessoa JCS. Sistema de Informação em Saúde: Gestão e Assistência no Sistema Único de Saúde. Cogitare Enferm. [Internet] 2014; 19(4) [acesso em 26 jan 2016]. Disponível: http://dx.doi.org/10.5380/ce.v19i4.35347.

25. Rehem TCMSB, de Oliveira MRF, Ciosak SI, Egry EY. Registro das internações por condições sensíveis a atenção primária: validação do sistema de informação hospitalar. Rev. Latino-Am. Enfermagem 2013; 21(5): 1-6.

26. Ministério da Saúde (BR). Secretaria de Vigilância em Saúde. Programa Nacional de DST e Aids. Protocolo para a prevenção de transmissão vertical de HIV e sífilis: Manual de Bolso. Brasília: Ministério da Saúde; 2007.

27. Ministério da Saúde (Brasil). Secretaria de Vigilância em Saúde. Departamento de Vigilância Epidemiológica. Manual de Rede de Frio. 4ª ed. [Internet] Brasília (DF): Ministério da Saúde; 2013 [acesso em 26 jan 2016]. Disponível: http://pni.datasus.gov.br/Download/manual_rede_frio.pdf.

28. Nakano AR, Bonan C, Teixeira LA. A normalização da cesárea como modo de nascer: cultura material do parto em maternidades privadas no Sudeste do Brasil. Physis. [Internet] 2015; 25(3) [acesso em 26 jan 2016]. Disponível: http://dx.doi.org/10.1590/S0103-73312015000300011.

29. Bittencourt F, Vieira JB, de Almeida ACCH. Concepção de gestantes sobre o parto cesariano. Cogitare Enferm. [Internet] 2013; 18(3) [acesso em 26 jan 2016]. Disponível: http://dx.doi.org/10.5380/ce.v18i3.33565. 